\title{
Low sex hormone-binding globulin is associated with hypertension: a cross-sectional study in a Swedish population
}

\author{
Bledar Daka ${ }^{1}$, Thord Rosen ${ }^{2}$, Per Anders Jansson ${ }^{3}$, Charlotte A Larsson ${ }^{4}$, Lennart Råstam ${ }^{4}$ and Ulf Lindblad ${ }^{\text {* }}$
}

\begin{abstract}
Background: The aim of this study was to investigate the association of sex hormone-binding globulin (SHBG) and hypertension in a Swedish population.

Methods: The study is based on a random sample of a Swedish population of men and women aged 30-74 years $(n=2,816)$. Total testosterone, oestradiol and SHBG were measured in 2,782 participants. Free androgen index was then calculated according to the formula FAI $=100 \times$ (Total testosterone)/SHBG. Hypertension was diagnosed according to JNC7.

Results: In men, but not in women, significant association between SHBG and both diastolic (diastolic blood pressure: $\beta=-0.143 p<0.001$ ) and systolic blood pressure (systolic blood pressure $\beta=-0.114 p<0.001$ ) was found. The association was still significant after adjusting for age, body mass index (BMI), homeostatic model assessment insulin resistance (HOMA-IR), triglycerides, high density lipoproteins (HDL) and C-reactive protein (CRP) (diastolic blood pressure: $\beta=-0.113 p<0.001$; systolic blood pressure $\beta=-0.093 p=0.001$ ). An inverse association was observed between SHBG and hypertension in both men $(B=-0.024 p<0.001)$ and women $(B=-0.022 p<0.001)$. The association was still significant in women older than 50 years after adjustments for age, BMI, physical activity, CRP and alcohol consumption ( $\mathrm{B}=-0.014, \mathrm{p}=0.008)$.

Conclusion: In conclusion, these results show a strong association between SHBG and blood pressure independent of major determinants of high blood pressure. This association might be addressed to direct effects of SHBG in endothelial cells through the receptor for SHBG. If this is confirmed by other observational and experimental studies, it might become a new field for the development of therapies for lowering blood pressure.
\end{abstract}

Keywords: Sex hormone binding globulin (SHBG), Testosterone, Gender, Hypertension, BMI

\section{Background}

Low concentrations of total testosterone in men have previously been associated with obesity, diabetes mellitus and hypertension, independent of age [1-6]. Studies in male rabbit models have shown that castration increases atherosclerosis [7]. The effects of testosterone on blood pressure levels may be explained by at least three factors. Firstly, testosterone is a vasodilatator in vitro, and in vivo experiments show a direct vasodilatation effect of testosterone [8]. Secondly, low concentrations of testosterone in men have

\footnotetext{
*Correspondence: ulf.lindblad@allmed.gu.se

'Department of Primary Health Care, Institute of Medicine, Gothenburg, Sweden

Full list of author information is available at the end of the article
}

been associated with higher total cholesterol and LDL-cholesterol and to lower levels of HDL-cholesterol, and consequently to atherosclerosis and hypertension [5,9-13]. Thirdly, an increased inflammatory response is associated with endothelial dysfunction and to an increased arterial stiffness and hypertension. Several clinical trials of testosterone replacement therapy in men with testosterone deficiency have shown a decrease in pro-inflammatory cytokines [14,15], indicating an immune-modulatory effect of testosterone. Nevertheless, the use of anabolic steroids (synthetic derivate of testosterone) is associated with hypertension [16].

A sizeable fraction of circulating testosterone is bound to sex hormone-binding globulin (SHBG); however, it is

\section{Biomed Central}

(c) 2013 Daka et al.; licensee BioMed Central Ltd. This is an Open Access article distributed under the terms of the Creative Commons Attribution License (http://creativecommons.org/licenses/by/2.0), which permits unrestricted use, distribution, and reproduction in any medium, provided the original work is properly cited. 
still not established if the observed link between the total testosterone and several cardiovascular risk factors (atherogenic lipid profile, type 2 diabetes, obesity) mirrors an effect of free circulating testosterone, or whether SHBG plays a more direct role. A recent study by Ding et al. [17] showed a strong association of SHBG as well as genes controlling the expression of SHBG with the risk for type 2 diabetes, but not between free testosterone and type 2 diabetes. While these results are supported by other authors [18], the role of SHBG in the development of type 2 diabetes is still subject to discussions and its contribution to the development of hypertension has to our knowledge not been investigated at all.

The purpose of this study was therefore to investigate the association between hypertension and SHBG, in both men and women in a Swedish population.

\section{Methods}

Between 2001 and 2005 a random sample of subjects, aged 30-74 years residing in two municipalities in South-western Sweden, were enrolled in the study [19]. All individuals that responded to the study invitation and gave written and informed consent were included as participants if they also contributed with physical examination, filling in the questionnaire, and by donating venous blood. Participants were stratified by gender in 5-year age groups. In Vara, 1,811 participants were enrolled (81\% participation rate), while the survey in Skövde included 1,005 subjects (70\%), with the overall participation rate being $76 \%$. After exclusion of subjects without successful analyses for SHBG, 2,782 subjects (Men=1,385; Women=1,397) remained to be analysed.

\section{Medical history, socio-economic and life style factors}

Details on the study protocol have been published previously [19]. Standard questionnaires were used to gain information on previous hospitalizations, medication (including preventive hormonal medication and post menopausal hormonal medication), smoking and alcohol habits as well as leisure time physical activity (LTPA). Standard instruments were also used for the collection of data on demographic and socio-economic factors such as social networks, social stress, and symptoms of anxiety and depression.

LTPA was characterised based on the response of questionnaire item: 'How much physical activity do you engage in during your leisure time?' [20] The question referred to the past year and the answer alternatives were: 1. Sedentary leisure time: Reading, TV, stamp collecting or other sedentary activity; 2. Light LTPA: Walking, cycling or other physical activity under at least four hours per week; 3. Moderate LTPA: Running, swimming, tennis, aerobic, heavier gardening or similar physical activity during at least 2 hours a week; and 4. Heavy training or competitive sport: Heavy training or competitions in running, skiing, swimming, football, etc. performed regularly and several times per week. Smoking habits was defined as current, former or daily smoking. Alcohol consumption was assessed by a standardized set of questions on how many days during the last 30 days had the subjects consumed beer, wine and strong liquor, respectively. Each of these questions was followed by questions on how many cans, glasses and/or bottles were normally consumed on such days. The total gram of alcohol consumed per week was then calculated by multiplying the number of days of alcohol drinking with the gram of alcohol that the items of consumed alcoholic beverage contain [21].

\section{Physical examination}

The nurses measured the standard resting blood pressure twice, one minute apart after five minutes rest with the subjects in a supine position. (Right brachial artery, arm at heart level, cuff size adjusted for arm circumference and reading the pressure at the closest $2 \mathrm{mmHg}$ ). The mean value of the two blood pressure readings was used for the analyses.

\section{Clinical chemistry}

Samples including plasma and serum were drawn after an overnight fasting and were immediately frozen at $-82^{\circ} \mathrm{C}$. In participants without known diabetes mellitus (DM), an OGTT was performed. Total cholesterol, HDL-cholesterol, LDL-cholesterol and S-triglycerides were analysed using standard procedures.

Total testosterone was analysed with kits Beckman Coulter 2003, 386982A (CV=7-8\%) and estradiol (CV=9$15 \%)$ and sex hormone-binding globulin (SHBG) $(\mathrm{CV}=5 \%)$ were analysed by kits Siemens Immulite 2000XPi [22]. These analyses were conducted in Malmö (Skåne University Hospital) and at Unilabs at Skaraborg Hospital in Skövde with results expressed in nmol/L. In 19 cases, the SHBG concentrations were reported to be higher than $180 \mathrm{nmol} / \mathrm{L}$; however, they were not further specified. Thus, we assumed those to be $180 \mathrm{nmol} / \mathrm{L}$. We calculated a free androgen index (FAI) for the estimation of free testosterone by the formula FAI=100xTotal testosterone/SHBG [23].

\section{Phenotypic characterisation}

Impaired fasting glucose, impaired glucose tolerance and diabetes mellitus were defined according to the WHO criteria [24]. Hypertension was defined according to the JNC 7 criteria [25]. BMI was calculated using the formula $B M I=w e i g h t(k g) / h i g h(m)^{2}$. Insulin resistance was estimated using the homeostatic model assessment for insulin resistance (HOMA-IR) [26].

\section{Ethical considerations}

All participants gave signed informed consent before being enrolled in the study, and the Ethical Committee 
at the University of Gothenburg, Sweden, approved the study.

\section{Statistics}

Standard methods were used for descriptive statistics. The association between SHBG, sex hormones and blood pressure was investigated in simple and multiple linear regression models after exclusion of subjects treated with blood pressure lowering drugs. Logistic regression analyses were performed to estimate the association between hypertension and sex hormones-SHBG. Associations were expressed as regression coefficients (B) and odds ratios (OR), respectively, both with $95 \%$ confidence intervals (CI). General linear models were used to estimate differences (CI) between continuous variables. Theoretical multivariate models were used to estimate the role of possible confounders on the investigated association. All analyses were two-sided and the significance was accepted if $\mathrm{p}<0.05$. All analyses were performed using SPSS Statistics for Mac. In order to evaluate the strength of the association for each possible risk factors of hypertension, we standardised the variables in consideration by the formula $\mathrm{SV}=\mathrm{V} / \mathrm{SD}$, where $\mathrm{SV}$ is the standardised variable. $\mathrm{V}$ is the value of the variable and $\mathrm{SD}$ is the standard deviation. We then ranked their association with hypertension by the regression coefficient $\beta$ in the logistic regression analyses.

\section{Results}

The characteristics of the study population are presented in Table 1. In men, the SHBG increased linearly with age but in women there was a u-shaped association between age and SHBG. A strong negative association between SHBG and BMI, fasting plasma glucose, HOMA-IR and TG was found in both men and women and the same was true for systolic and diastolic blood pressure. SHBG was strongly associated with total testosterone in men and oestradiol in women.

In our population $271(10 \%)$ individuals were under treatment for hypertension. The association between SHBG and blood pressure after excluding subjects on medication for hypertension is presented in Table 2. In men, significant association between SHBG and both diastolic (diastolic blood pressure: $\beta=-0.143 \mathrm{p}<0.001$ ) and systolic blood pressure (systolic blood pressure $\beta=-0.114$ $\mathrm{p}<0.001$ ) was found. The association was still significant after adjusting for age, BMI, HOMA-IR, triglycerides, HDL and CRP (diastolic blood pressure: $\beta=-0.113$ $\mathrm{p}<0.001$; systolic blood pressure $\beta=-0.093 \mathrm{p}=0.001$ ). In women, no association between SHBG and systolic and diastolic blood pressure, respectively, was found.

Age-adjusted differences of SHBG concentrations in different blood pressure categories in accordance with JNC7 are presented in Figure 1. In men, SHBG decreased significantly with the severity of high blood pressure. In women, however, the SHBG was almost constant in categories NT and prehypertension1-3. Hypertensive women had significantly lower concentrations of SHBG when compared with all other categories $(\mathrm{p}<0.001)$.

The associations between quartiles of SHBG and hypertension are presented in Table 3 for both men and

Table 1 Phenotypical characterisation of sex hormone-binding globulin in a Swedish population of men and women

\begin{tabular}{|c|c|c|c|c|c|c|c|c|c|c|c|c|}
\hline & \multicolumn{6}{|l|}{ Men 1,385 } & \multicolumn{6}{|c|}{ Women 1,397 } \\
\hline & \multirow[b]{2}{*}{ All } & \multicolumn{4}{|c|}{ Quartiles for SHBG } & \multirow[t]{2}{*}{$p$-trend } & \multirow[b]{2}{*}{ All } & \multicolumn{4}{|c|}{ Quartiles for SHBG } & \multirow[t]{2}{*}{$p$-trenc } \\
\hline & & Q1 & Q2 & Q3 & Q4 & & & Q1 & Q2 & Q3 & Q4 & \\
\hline SHBG & $33 \pm 14$ & 17.8 & 26.7 & 35.1 & 50.8 & & $55 \pm 31$ & 26.2 & 41.1 & 55.6 & 92.6 & \\
\hline Age & $47.8 \pm 11.8$ & 42.2 & 45.6 & 48.8 & 54.4 & $<0.001$ & $47.7 \pm 11.7$ & 46.9 & 47.3 & 49.0 & 47.7 & 0.033 \\
\hline BMl & $26.9 \pm 3.6$ & 28.9 & 27.3 & 26.5 & 24.9 & $<0.001$ & $26.8 \pm 5.3$ & 30.1 & 27.2 & 26.5 & 24.9 & $<0.001$ \\
\hline Fasting insulin & $6.7 \pm 5.2$ & 8.9 & 7.0 & 6.2 & 4.9 & $<0.001$ & $6.2 \pm 4.4$ & 8.5 & 6.2 & 5.1 & 4.7 & $<0.001$ \\
\hline Fasting glucose & $5.5 \pm 1.1$ & 5.8 & 5.7 & 5.5 & 5.4 & $<0.001$ & $5.3 \pm 1.1$ & 5.7 & 5.4 & 5.1 & 5.1 & $<0.001$ \\
\hline HOMA-IR & $1.7 \pm 1.6$ & 2.3 & 1.8 & 1.5 & 1.1 & $<0.001$ & $1.5 \pm 1.3$ & 2.2 & 1.5 & 1.2 & 1.1 & $<0.001$ \\
\hline Hs-CRP & $2.4 \pm 5.8$ & 3.0 & 2.3 & 1.9 & 3.3 & 0.038 & $2.7 \pm 4.6$ & 4.1 & 2.5 & 2.2 & 2.3 & 0.097 \\
\hline LDL & $3.4 \pm 0.9$ & 3.4 & 3.4 & 3.4 & 3.3 & 0.327 & $3.1 \pm 0.9$ & 3.2 & 3.1 & 3.2 & 3.2 & 0.287 \\
\hline $\mathrm{HDL}$ & $1.2 \pm 0.3$ & 1.1 & 1.2 & 1.2 & 1.3 & $<0.001$ & $1.4 \pm 0.3$ & 1.2 & 1.4 & 1.5 & 1.5 & $<0.001$ \\
\hline Triglycerides & $1.5 \pm 0.9$ & 1.9 & 1.5 & 1.3 & 1.1 & $<0.001$ & $1.2 \pm 0.6$ & 1.4 & 1.1 & 1.0 & 1.1 & 0.004 \\
\hline Systolic BP & $123 \pm 16$ & 126 & 124 & 123 & 123 & $<0.001$ & $119 \pm 18$ & 123 & 118 & 117 & 119 & 0.008 \\
\hline Diastolic BP & $72 \pm 10$ & 73.8 & 71.7 & 71.8 & 70.6 & $<0.001$ & $69 \pm 10$ & 70.0 & 68.4 & 67.8 & 68.2 & 0.007 \\
\hline Total Testosterone & $14.3 \pm 4.4$ & 11.2 & 13.4 & 15.2 & 17.5 & $<0.001$ & $1.3 \pm 1.3$ & 1.3 & 1.2 & 1.2 & 1.3 & 0.853 \\
\hline Oestradiol & $127 \pm 56$ & 125 & 128 & 124 & 132 & 0.026 & $320 \pm 396$ & 255 & 328 & 337 & 363 & $<0.001$ \\
\hline FAI & $48.5 \pm 17.3$ & 64.5 & 50.5 & 43.4 & 35.3 & $<0.001$ & $3.1 \pm 5.8$ & 5.7 & 2.9 & 2.4 & 1.4 & $<0.001$ \\
\hline
\end{tabular}

SHBG = sex hormone-binding globulin, Q1 is the lowest quartile and Q4 is the highest. BMI=Body mass index, HOMA-IR= homeostatic model assessment insulin resistance, $\mathrm{hs}-\mathrm{CRP}=$ high sensitive $\mathrm{c}$-reactive protein, $\mathrm{LDL}=$ low density lipoprotein, $\mathrm{HDL}=$ high density protein, $\mathrm{BP}=\mathrm{blood}$ pressure, $\mathrm{FAl}=$ free androgen index. General linear models to estimate mean values and differences between quartiles were used. The significance was estimated with $p$-value. 
Table 2 The association between total testosterone, SHBG, oestradiol, free testosterone, respectively, and blood pressure in men and women

\begin{tabular}{|c|c|c|c|c|c|c|c|c|}
\hline & \multicolumn{4}{|c|}{ Men 1,255 } & \multicolumn{4}{|c|}{ Women 1,259 } \\
\hline & \multicolumn{2}{|c|}{ Systolic blood pressure } & \multicolumn{2}{|c|}{ Diastolic blood pressure } & \multicolumn{2}{|c|}{ Systolic blood pressure } & \multicolumn{2}{|c|}{ Diastolic blood pressure } \\
\hline & $\beta$ & $\mathrm{p}$ & $\beta$ & $P$ & $\beta$ & $\mathrm{p}$ & $\beta$ & $\mathbf{P}$ \\
\hline \multicolumn{9}{|l|}{ Model 1 Adjusted for age } \\
\hline Total testosterone & -0.136 & $<0.001$ & -0.121 & $<0.001$ & -0.011 & 0.470 & -0.013 & 0.638 \\
\hline Sex hormone-binding globulin & -0.114 & $<0.001$ & -0.143 & $<0.001$ & -0.031 & 0.193 & -0.055 & 0.041 \\
\hline Oestradiol & -0.014 & 0.560 & 0.022 & 0.408 & -0.035 & 0.144 & -0.057 & 0.036 \\
\hline Free testosterone & -0.051 & 0.119 & -0.051 & 0.154 & 0.009 & 0.698 & 0.016 & 0.574 \\
\hline \multicolumn{9}{|l|}{ Model 2 Adjusted for age and BMI } \\
\hline Total testosterone & -0.114 & $<0.001$ & -0.098 & 0.001 & -0.008 & 0.873 & -0.018 & 0.576 \\
\hline Sex hormone-binding globulin & -0.083 & 0.005 & -0.117 & $<0.001$ & 0.020 & 0.685 & -0.018 & 0.610 \\
\hline Oestradiol & -0.019 & 0.440 & 0.017 & 0.530 & -0.036 & 0.121 & -0.025 & 0.461 \\
\hline Free testosterone & -0.039 & 0.243 & -0.035 & 0.328 & -0.016 & 0.570 & -0.001 & 0.988 \\
\hline \multicolumn{9}{|c|}{ Model 3 Adjusted for age, BMI, TG, HDL and CRP } \\
\hline Total testosterone & -0.111 & $<0.001$ & -0.089 & 0.002 & -0.002 & 0.932 & -0.014 & 0.468 \\
\hline Sex hormone-binding globulin & -0.078 & 0.009 & -0.105 & 0.001 & 0.043 & 0.146 & 0.004 & 0.908 \\
\hline Oestradiol & -0.026 & 0.295 & 0.000 & 0.999 & -0.008 & 0.768 & -0.020 & 0.556 \\
\hline Free testosterone & -0.034 & 0.348 & -0.033 & 0.363 & -0.019 & 0.487 & -0.006 & 0.855 \\
\hline \multicolumn{9}{|c|}{ Model 4 Adjusted for age, HOMA-IR, Tg, HDL and CRP } \\
\hline Total testosterone & -0.123 & $<0.001$ & -0.097 & 0.001 & 0.005 & 0.846 & -0.008 & 0.814 \\
\hline Sex hormone-binding globulin & -0.093 & 0.001 & -0.113 & $<0.001$ & 0.041 & 0.166 & 0.013 & 0.718 \\
\hline Oestradiol & -0.028 & 0.277 & 0.000 & 0.994 & -0.004 & 0.902 & -0.017 & 0.611 \\
\hline Free testosterone & -0.035 & 0.307 & -0.032 & 0.878 & -0.014 & 0.620 & -0.005 & 0.876 \\
\hline
\end{tabular}

BMI: Body mass index, Tg: triglycerides, HOMA-IR: homeostatic model assessment insulin resistance, HDL: high density lipoprotein, CRP: c-reactive protein, $\beta$ : regressions coefficient, $p: p$ value. Linear regression analysis is used to investigate the association between hormonal levels and blood pressure. All subjects with known hypertension (271) were excluded from the analyses.

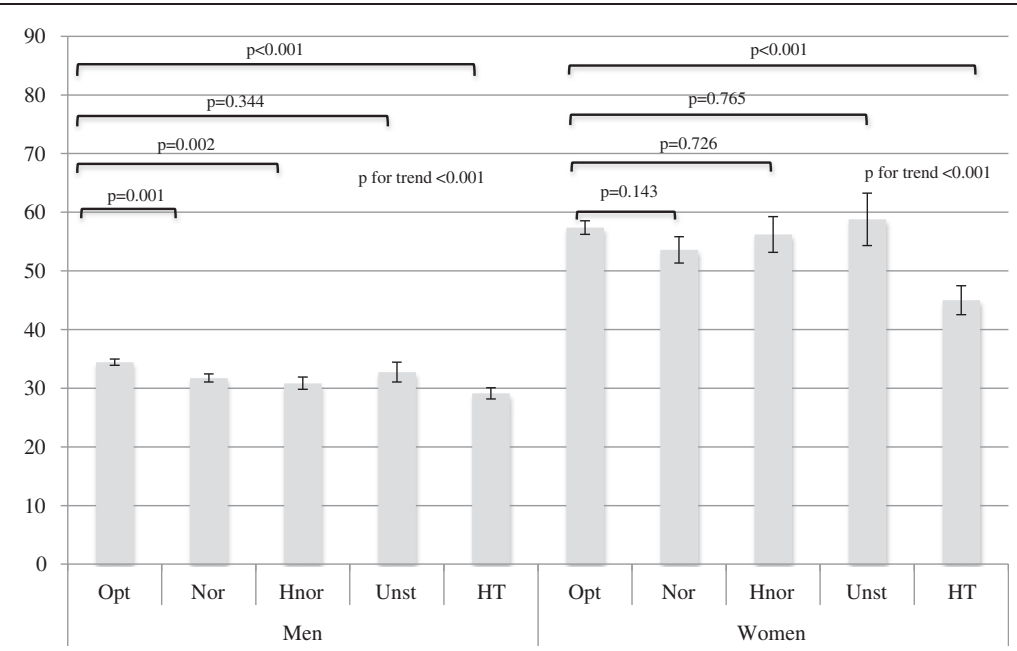

Figure 1 Comparison of serum concentrations of sex hormone-binding globulin ( $y$-axis) between different blood pressure categories. JNC7 blood pressure categories; Opt=normal optimal BP $<120 / 80$ mm Hg, Nor= normal BP $<130 / 85$ mm Hg, Hnor= normal high BP $<140 / 90$ mm Hg, Unst= unstable blood pressure Hypertension was defined as known documented diagnosis for high blood pressure, or by three consecutive BP reading $\geq 140 / 90 \mathrm{~mm} \mathrm{Hg}$ (systolic and/or diastolic). When the BP exceeded these limits only once or twice the BP was categorized as unstable. 
Table 3 Multivariate analyses of OR for hypertension associated with quartiles of sex hormone-binding globulin in women and men, respectively

\begin{tabular}{|c|c|c|c|c|c|c|c|c|}
\hline Men & & OR & $\mathrm{Cl}$ & $\mathbf{P}$ & Women & OR & $\mathrm{Cl}$ & $\mathbf{P}$ \\
\hline \multicolumn{9}{|c|}{ Model 1 Age adjusted OR for HYPERTENSION } \\
\hline \multirow[t]{3}{*}{ All } & Q 1 vs 4 & 2.2 & $1.24-3.75$ & 0.007 & All & 4.2 & $2.45-7.06$ & $<0.001$ \\
\hline & Q 2 vs 4 & 1.7 & $1.05-2.78$ & 0.031 & & 2.3 & $1.31-3.91$ & 0.004 \\
\hline & Q 3 vs 4 & 1.2 & $0.72-1.82$ & 0.549 & & 1.9 & $1.12-3.26$ & 0.017 \\
\hline \multirow[t]{3}{*}{$<50 \mathrm{y}$} & Q 1 vs 4 & 1.9 & $0.76-4.68$ & 0.174 & $<50 y$ & 2.8 & $1.14-7.22$ & 0.025 \\
\hline & Q 2 vs 4 & 1.6 & $0.61-4.24$ & 0.333 & & 1.7 & $0.61-4.49$ & 0.317 \\
\hline & Q 3 vs 4 & 0.7 & $0.25-2.18$ & 0.582 & & 1.6 & $0.53-4.98$ & 0.403 \\
\hline \multirow[t]{3}{*}{$>50 \mathrm{y}$} & Q 1 vs 4 & 2.1 & $0.99-4.66$ & 0.053 & $\geq 50 y$ & 4.8 & $2.61-8.88$ & $<0.001$ \\
\hline & Q 2 vs 4 & 1.6 & $0.92-2.78$ & 0.095 & & 2.3 & $1.21-4.22$ & 0.010 \\
\hline & Q 3 vs 4 & 1.2 & $0.70-1.95$ & 0.546 & & 2.1 & $1.17-3.82$ & 0.013 \\
\hline \multicolumn{9}{|c|}{ Model 2 Age and BMI adjusted OR for hypertension } \\
\hline \multirow[t]{3}{*}{ All } & Q 1 vs 4 & 1.5 & $0.82-2.67$ & 0.193 & $\geq 50 \mathrm{y}$ & 3.1 & $1.54-6.04$ & 0.001 \\
\hline & Q 2 vs 4 & 1.3 & $0.81-2.22$ & 0.250 & & 1.9 & $1.00-3.79$ & 0.049 \\
\hline & Q 3 vs 4 & 1.0 & $0.63-1.61$ & 0.982 & & 2.2 & $1.20-4.15$ & 0.012 \\
\hline \multicolumn{9}{|c|}{ Model 3 Age, BMI, smoking habits, hs-CRP, LDL, triglycerides, and alcohol consumption adjusted } \\
\hline \multirow[t]{3}{*}{ All } & Q 1 vs 4 & 1.5 & $0.78-2.70$ & 0.235 & $\geq 50$ y & 3.0 & $1.47-6.33$ & 0.003 \\
\hline & Q 2 vs 4 & 1.4 & $0.81-2.22$ & 0.217 & & 2.1 & $1.04-4.09$ & 0.038 \\
\hline & Q 3 vs 4 & 1.0 & $0.59-1.57$ & 0.867 & & 2.1 & $1.12-4.04$ & 0.021 \\
\hline \multicolumn{9}{|c|}{ Model 4 Age, HOMA-IR, smoking habits, hs-CRP, LDL, triglycerides, and alcohol consumption adjusted } \\
\hline \multirow[t]{3}{*}{ All } & Q 1 vs 4 & 1.8 & $0.96-3.27$ & 0.067 & $\geq 50 y$ & 2.7 & 1.33-5.60 & 0.006 \\
\hline & Q 2 vs 4 & 1.4 & $0.83-2.43$ & 0.202 & & 1.9 & $0.95-3.67$ & 0.070 \\
\hline & Q 3 vs 4 & 1.1 & $0.67-1.79$ & 0.721 & & 1.8 & $0.97-3.41$ & 0.061 \\
\hline
\end{tabular}

BMI: Body mass index, Tg: triglycerides, HOMA-IR: homeostatic model assessment insulin resistance, LDL: Low density lipoprotein, CRP: c-reactive protein, HRT: hormone replacement therapy, OR: odds ration for hypertension, p: p value. Logistic regression analysis is used to investigate the association between hormonal levels and hypertension. Possible confounders were taken into consideration in different models stepwise.

women. As observed, in women the odds for hypertension were higher for the lowest quartile $(\mathrm{OR}=4.2 \mathrm{p}<0.001)$. The association was different when comparing women under or over age 50 years (Women under $50 \mathrm{OR}=2.8$, CI 1.147.22, $\mathrm{p}=0.025$, women over $50 \mathrm{OR}=4.8$, CI 2.61-8.88, $\mathrm{p}<0.001)$. In women under 50 , the association was not significant when adjusting for age and BMI. In contrary, for women over 50, the association remained significant when we adjusted for age, HOMA-IR, smoking habits, hs-CRP, LDL, Tg, hormone-replacement therapy and alcohol consumption ( $\mathrm{p}$-trend $=0.05$ ). The association remained significant even when we included a known history of stroke and diabetes in the equation ( $p$-trend=0,043). In men, an association between quartiles of SHBG and hypertension $(\mathrm{OR}=2.2 \mathrm{p}=0.007)$ was found. The association was not significant when adjusting for BMI (Table 3).

In Table 4 we ranked age, HOMA-IR, BMI and SHBG by regression coefficient in order to evaluate the strength of the association with hypertension. Age was strongest associated with hypertension and BMI was second in both men and women. The association between SHBG and hypertension was stronger than the association between HOMA-IR (surrogate of insulin resistance) and hypertension in men but not in women.

The interaction between sex hormones, SHBG and hypertension was investigated in a logistic regression model, but no significant interaction was found. The interaction between age and SHBG in the hypertension and blood pressure was also investigated with regression models, but no significant difference was found.

\section{Discussion}

In this cross-sectional study, a strong inverse association between blood pressure and SHBG was observed. Postmenopausal women with hypertension had significantly lower SHBG concentrations, and this association was significant even after adjusting for major risk factors for hypertension such as age, BMI, diabetes and insulin resistance. In men, we observed an inverse association between systolic blood pressure and SHBG. This association was independent of major confounders. Both these findings indicate that low concentrations of SHBG may have independent negative effects in the control of blood pressure.

To our knowledge this is the first study to estimate the specific role of SHBG in blood pressure control. 
Table 4 Association between hypertension and sex hormone-binding globulin when major risk factors for hypertension are considered

\begin{tabular}{|c|c|c|c|c|c|c|c|c|}
\hline Men & & $\beta$ & $\mathbf{p}$ & Exp & Women & $\beta$ & $p$ & Exp \\
\hline \multirow[t]{4}{*}{ All } & Age & 1.352 & $<0.001$ & 3.9 & & 1.299 & $<0.001$ & 3.7 \\
\hline & $B M I$ & 0.298 & 0.004 & 1.3 & & 0.376 & 0.002 & 1.5 \\
\hline & HOMA-IR & 0.060 & 0.497 & 1.1 & & 0.347 & 0.002 & 1.4 \\
\hline & $S H B G$ & -0.194 & 0.061 & 0.8 & & -0.297 & 0.027 & 0.7 \\
\hline \multirow[t]{4}{*}{$<50$} & Age & 0.619 & $<0.001$ & 1.9 & & 0.967 & $<0.001$ & 2.6 \\
\hline & $B M I$ & 0.298 & 0.004 & 1.3 & & 0.532 & 0.012 & 1.7 \\
\hline & HOMA-IR & 0.049 & 0.497 & 1.1 & & 0.018 & 0.927 & 1.0 \\
\hline & $S H B G$ & -0.164 & 0.061 & 0.8 & & -0.103 & 0.698 & 0.9 \\
\hline \multirow[t]{4}{*}{$\geq 50$} & Age & 0.882 & $<0.001$ & 2.4 & & 0.723 & $<0.001$ & 2.1 \\
\hline & $B M I$ & 0.298 & 0.004 & 1.3 & & 0.382 & 0.012 & 1.5 \\
\hline & HOMA-IR & 0.072 & 0.491 & 1.1 & & 0.538 & 0.001 & 1.7 \\
\hline & $S H B G$ & -0.210 & 0.063 & 0.8 & & -0.333 & 0.033 & 0.7 \\
\hline
\end{tabular}

BMI: Body mass index, HOMA-IR: homeostatic model assessment insulin resistance, SHBG: sex hormone-binding globulin, $\beta$ : regressions coefficient, $\mathrm{p}$ : $\mathrm{p}$ value, Exp: the odds change for hypertension for increasing with 1 standard deviation of variable. Age, BMI, HOMA-IR are standardised in order to compare the impact in hypertension. Logistic regression analysis is used to rank the strength of the association. All women with hormonal therapy are excluded from the analyses. Every considered variable is standardised. The purpose was to rank the strength of the association with hypertension for each variable and the value of $\beta$ decide the strength of the association. We standardised the variables in consideration by the formula SV=V/SD, where SV is the standardised variable. $\mathrm{V}$ is the value of the variable and SD is the standard deviation.

There are some publications concerning the association between hypertension and SHBG in men, but very few in women. The majority of studies, aimed to estimate the effects of testosterone on blood pressure in men, provided information about the level of SHBG. This was done in order to estimate the concentrations of free testosterone. However, only a few studies have presented information about the association between SHBG and hypertension. In a prospective study in men, aimed to investigate the role of sex hormones in hypertension, Khaw et al. [5] observed that total testosterone but not SHBG was associated with hypertension. However, in accordance with our results, they found an inverse association between SHBG and diastolic blood pressure adjusted for age. The definition of hypertension in that study was different from our definition based on JNC7 (160/95 in Rancho Bernard and 140/90 in VSC). A cross-sectional study in men conducted in Tromsö [27], which aimed to investigate the association between testosterone on one hand and hypertension and left ventricular hypertrophy on the other hand, found an age-independent association between SHBG and both systolic and diastolic blood pressure. The association remained borderline significant for the systolic blood pressure $(\mathrm{p}=0,057)$ even after adjusting for BMI and alcohol consumption, but for diastolic blood pressure it was not significant $(\mathrm{p}=0,370)$. Significantly lower concentrations of SHBG were found in hypertensive men even after adjusting for age, BMI and SHBG concentrations in accordance with our results in women [27].

Some gender differences were, however observed in our study (Figure 1). In accordance with Khaw et al. [5] levels of SHBG decreased in a linear fashion in men when the blood pressure increased. In contrary in women the decrease in SHBG were firstly observed when they had developed hypertension. As the association between SHBG and hypertension in women was stronger over the age of 50 we suppose that these gender differences can at least in part be addressed to the menopause. In a prospective study including men and post-menopausal women, Ding et al. [17] observed a predictive value of SHBG concentrations concerning incident cases of type 2 diabetes, in both men and women. They reported an association between hypertension and low concentrations of SHBG in women in the crude analysis. However, the authors did not report whether any multivariate statistical tests accounting for possible confounders were computed. Neither was the association between SHBG and hypertension in males at baseline reported.

We did not found studies comparing the strength of the association between SHBG and hypertension with other risk factors for hypertension. In order to compare the SHBG with other conventional risk factors for hypertension, we standardized age, BMI, HOMA-IR and SHBG. In our study a weak association between insulin resistance and hypertension was observed in men were SHBG had stronger association with hypertension than HOMA-Ir.

Tot- $\mathrm{T}$ but not free testosterone was associated with hypertension in our population. Testosterone deficiency has been indicated as a risk factor for obesity and cardiovascular diseases. The question as to whether $\mathrm{T}$ has a direct effect on blood vessels remains to be investigated. We cannot confirm the vasodilatative effects of free testosterone that were shown by Webb et al. [8]. Our results suggest 
that the association between SHBG and blood pressure may explain the association between testosterone and blood pressure, at least partially. A recent study has shown that SHBG but not testosterone is associated with increasing of blood pressure [28]. In the matter of diabetes risk, Lakshman et al. [18] speculate on a modulatory effect of SHBG that enhances the effects of testosterone. In fact, a membrane receptor for SHBG is present in uterine endometrial cell membranes, isolated prostatic cell membranes, human placenta, normal breast, liver and epididymis but not in striated muscle [29]. This receptor, when activated after contact with SHBG, can stimulate the production of c-AMP. It must be emphasized that in a minor study with men with hypertension it was found a strong association between SHBG and renin that can be another pathway for direct effects of SHBG on the control of blood pressure [30]. Whether this association occurs in the blood vessel and if it influences the blood pressure remains to be confirmed by studies of a different design. For example, experimental studies on mice with knocked gene for SHBG could help in understanding the functions of the protein, and determine whether this protein only has carrying functions or if the functions of the protein are extended inside the target cells for androgen effect.

In accordance with previous studies, we found a positive linear association between SHBG and age in men while in women the association is inverse before 50 years of age $[31,32]$. The mechanisms lying behind this gender duality are not fully understood. Some authors suggest that in men a decrease of testosterone concentrations due to age is the cause of increasing SHBG concentrations as testosterone is supposed to have an inhibitory effect on SHBG production. Such an effect does not seem to be determinant in increasing SHBG concentrations in aging men according to De Ronde et al. [33].

\section{Strengths}

This was a large population based study that included both men and women. Therefore, we could compare the association of oestradiol, SHBG and testosterone in both men and women. The study had a high participation rate of $76 \%$, including a younger middle-aged population. The diagnosis of hypertension was précised with the trained nurse. In this large study population, we also had information about the medication influencing the blood pressure that gave us the possibility to exclude subjects with blood pressure lowering medication or with hormonal medication that could have influenced the analyses. Blood samples were collected in the morning, thereby avoiding influences of circadian changes in the hormonal concentrations.

\section{Limitations}

A potential weakness of this study might be the lack of information with regard to menopausal status. Still, the use of
50 years as cut-off point for menopause is supported by findings from previous studies $[34,35]$. It should also be emphasized that as this is a cross-sectional study the causality between SHBG and hypertension cannot be established and it is possible that hypertension or associated factors lead to decreased concentrations of sex hormonebinding globulin. Another limitation of the study is the low accuracy of the measurements of testosterone in the low range of the distribution thus concerns especially women [36]. Moreover there is a circadian variation of concentrations of testosterone and SHBG which probably dilute our findings [37].

\section{Conclusion}

These results show a strong association between SHBG and blood pressure independent of the components of metabolic syndrome and inflammation. This association might be explained by a direct effect of SHBG in endothelial cells through the receptor for SHBG. If others confirm this it might become a new field for the development of therapies for lowering blood pressure.

\begin{abstract}
Abbreviations
SHBG: Sex hormone-binding globulin; FAl: Free androgen index; JNC: Joint National Committee on Detection, Evaluation and Treatment of High Blood Pressure; BMI: Body mass index; HOMA: IR-homeostatic model assessment of insulin resistance; CRP: c-reactive protein; HDL: High density lipoprotein; LDL: Low density lipoprotein; Tg: Triglycerides; LTPA: Leisure time physical activity; OGTT: Oral glucose tolerance test; WHO: World Health Organisation; OR: Odds ratio; Cl: Confidence interval; SD: Standard deviation.
\end{abstract}

\section{Competing interests}

The authors report no competing interest. The authors alone are responsible for the content and writing of this paper.

\section{Authors' contributions}

$\mathrm{BD}$ prepared the data, performed the statistical analyses, drafted the manuscript and took part in conceiving the study. TR and PAJ offered their expertise in endocrinology and metabolism questions. CAL worked on preparing data and offered expertise in statistical analysis. LR conceived the study and acquired the data. UL conceived and coordinated the study, as well as acquired the data. All authors took part in the design of the study, the interpretation of data, the revision of the manuscript, and read and approved the final manuscript.

\section{Acknowledgements}

This study was supported by grants from the Skaraborg Institute and the Research and Development Council of the Region Västra Götaland and we want to thank them for supporting us. We are also very thankful to every participant from Vara and Skövde that made our study possible giving his or her contribution.

\section{Funding}

This study was supported by grants from the Skaraborg Institute and the Research and Development Council of the Region Västra Götaland.

\section{Author details}

${ }^{1}$ Department of Primary Health Care, Institute of Medicine, Gothenburg, Sweden. ${ }^{2}$ Department of Endocrinology, Gothenburg, Sweden. ${ }^{3}$ Department of Internal Medicine, University of Gothenburg, PO Box 454, Gothenburg SE-405 30, Sweden. ${ }^{4}$ Department of Clinical Sciences, Malmö, Social Medicine and Global Health, CRC 28-12, Lund University, Jan Waldenströms gata 35, Malmö 205 02, Sweden. 
Received: 19 September 2012 Accepted: 17 March 2013 Published: 18 April 2013

\section{References}

1. Dandona P, Dhindsa S, Chaudhuri A, Bhatia V, Topiwala S: Hypogonadotrophic hypogonadism in type 2 diabetes. Aging Male 2008, 11(3):107-117.

2. Haffner SM: Sex hormone-binding protein, hyperinsulinemia, insulin resistance and noninsulin-dependent diabetes. Horm Res 1996, 45(3-5):233-237.

3. Oh JY, Barrett-Connor E, Wedick NM, Wingard DL: Endogenous sex hormones and the development of type 2 diabetes in older men and women: the Rancho Bernardo study. Diabetes Care 2002, 25(1):55-60

4. Haffner SM: Sex hormones, obesity, fat distribution, type 2 diabetes and insulin resistance: epidemiological and clinical correlation. Int J Obes Relat Metab Disord 2000, 24(Suppl 2):S56-58.

5. Khaw KT, Barrett-Connor E: Blood pressure and endogenous testosterone in men: an inverse relationship. J Hypertens 1988, 6(4):329-332.

6. Kienitz T, Quinkler M: Testosterone and blood pressure regulation. Kidney Blood Press Res 2008, 31(2):71-79.

7. Yue P, Chatterjee K, Beale C, Poole-Wilson PA, Collins P: Testosterone relaxes rabbit coronary arteries and aorta. Circulation 1995, 91(4):1154-1160

8. Webb CM, McNeill JG, Hayward CS, de Zeigler D, Collins P: Effects of testosterone on coronary vasomotor regulation in men with coronary heart disease. Circulation 1999, 100(16):1690-1696.

9. Barrett-Connor E: Lower endogenous androgen levels and dyslipidemia in men with non-insulin-dependent diabetes mellitus. Ann Intern Med 1992, 117(10):807-811.

10. Barrett-Connor E, Khaw KT: Endogenous sex hormones and cardiovascular disease in men. A prospective population-based study. Circulation 1988, 78(3):539-545.

11. Barud W, Palusinski R, Beltowski J, Wojcicka G: Inverse relationship between total testosterone and anti-oxidized low density lipoprotein antibody levels in ageing males. Atherosclerosis 2002, 164(2):283-288.

12. Kapoor D, Clarke S, Stanworth R, Channer KS, Jones TH: The effect of testosterone replacement therapy on adipocytokines and C-reactive protein in hypogonadal men with type 2 diabetes. Eur J Endocrinol 2007, 156(5):595-602.

13. Simon D, Charles MA, Nahoul K, Orssaud G, Kremski J, Hully V, Joubert E, Papoz L, Eschwege E: Association between plasma total testosterone and cardiovascular risk factors in healthy adult men: The Telecom Study. J Clin Endocrinol Metab 1997, 82(2):682-685.

14. Guler N, Batyraliev T, Dulger H, Ozkara C, Tuncer M, Aslan S, Okut H, Agirbasli M: The effects of short term (3 weeks) testosterone treatment on serum inflammatory markers in men undergoing coronary artery stenting. Int J Cardiol 2006, 109(3):339-343.

15. Musabak U, Bolu E, Ozata M, Oktenli C, Sengul A, Inal A, Yesilova Z, Kilciler G, Ozdemir IC, Kocar IH: Gonadotropin treatment restores in vitro interleukin-1 beta and tumour necrosis factor-alpha production by stimulated peripheral blood mononuclear cells from patients with idiopathic hypogonadotropic hypogonadism. Clin Exp Immunol 2003, 132(2):265-270.

16. Sullivan ML, Martinez CM, Gennis P, Gallagher EJ: The cardiac toxicity of anabolic steroids. Prog Cardiovasc Dis 1998, 41(1):1-15.

17. Ding EL, Song Y, Manson JE, Hunter DJ, Lee CC, Rifai N, Buring JE, Gaziano $J M$, Liu S: Sex hormone-binding globulin and risk of type 2 diabetes in women and men. N Engl J Med 2009, 361(12):1152-1163.

18. Lakshman KM, Bhasin S, Araujo AB: Sex hormone-binding globulin as an independent predictor of incident type 2 diabetes mellitus in men. J Gerontol A Biol Sci Med Sci 2010, 65(5):503-509.

19. Nyholm M, Gullberg B, Haglund B, Rastam L, Lindblad U: Higher education and more physical activity limit the development of obesity in a Swedish rural population. The Skaraborg Project. Int J Obes (Lond) 2008, 32(3):533-540

20. Booth ML, Owen N, Bauman A, Gore CJ: Relationship between a 14-day recall measure of leisure-time physical activity and a submaximal test of physical work capacity in a population sample of Australian adults. Res $Q$ Exerc Sport 1996, 67(2):221-227.

21. Goransson M, Hanson BS: How much can data on days with heavy drinking decrease the underestimation of true alcohol consumption? J Stud Alcohol 1994, 55(6):695-700.
22. Kamal S: Radioimmunoassay and related techniques. J Pak Med AssoC 1993, 43(11):224-225.

23. Morris PD, Malkin CJ, Channer KS, Jones TH: A mathematical comparison of techniques to predict biologically available testosterone in a cohort of 1072 men. Eur J Endocrinol 2004, 151(2):241-249.

24. Alberti KG, Zimmet PZ: Definition, diagnosis and classification of diabetes mellitus and its complications. Part 1: diagnosis and classification of diabetes mellitus provisional report of a WHO consultation. Diabet Med 1998, 15(7):539-553.

25. World Health Organization and International Society of Hypertension: Guidelines for the Management of Hypertension. J Hypertens 1999, 17:151-183.

26. Matthews DR, Hosker JP, Rudenski AS, Naylor BA, Treacher DF, Turner RC: Homeostasis model assessment: insulin resistance and beta-cell function from fasting plasma glucose and insulin concentrations in man. Diabetologia 1985, 28(7):412-419.

27. Svartberg J, von Muhlen D, Schirmer $\mathrm{H}$, Barrett-Connor E, Sundfjord J, Jorde R: Association of endogenous testosterone with blood pressure and left ventricular mass in men. The Tromso Study. Eur J Endocrinol 2004, 150(1):65-71.

28. Wang L, Szklo M, Folsom AR, Cook NR, Gapstur SM, Ouyang P: Endogenous sex hormones, blood pressure change, and risk of hypertension in postmenopausal women: the Multi-Ethnic Study of Atherosclerosis. Atherosclerosis 2012, 224(1):228-234.

29. Krupenko SA, Krupenko NI, Danzo BJ: Interaction of sex hormone-binding globulin with plasma membranes from the rat epididymis and other tissues. J Steroid Biochem Mol Biol 1994, 51(1-2):115-124.

30. Phillips GB, Jing TY, Laragh JH, Sealey JE: Serum sex hormone levels and renin-sodium profile in men with hypertension. Am J Hypertens 1995, 8(6):626-629.

31. Harman SM, Metter EJ, Tobin JD, Pearson J, Blackman MR: Longitudinal effects of aging on serum total and free testosterone levels in healthy men. Baltimore Longitudinal Study of Aging. J Clin Endocrinol Metab 2001, 86(2):724-731

32. Maggio M, Lauretani F, Basaria S, Ceda GP, Bandinelli S, Metter EJ, Bos AJ, Ruggiero C, Ceresini G, Paolisso G, et al: Sex hormone binding globulin levels across the adult lifespan in women-the role of body mass index and fasting insulin. J Endocrinol Invest 2008, 31(7):597-601.

33. de Ronde W, van der Schouw YT, Muller M, Grobbee DE, Gooren LJ, Pols $H A$, de Jong FH: Associations of sex-hormone-binding globulin (SHBG) with non-SHBG-bound levels of testosterone and estradiol in independently living men. J Clin Endocrinol Metab 2005, 90(1):157-162.

34. Jansen SC, Temme EH, Schouten EG: Lifetime estrogen exposure versus age at menopause as mortality predictor. Maturitas 2002, 43(2):105-112.

35. de Kleijn MJ, van der Schouw YT, Verbeek AL, Peeters PH, Banga JD, van der Graaf Y: Endogenous estrogen exposure and cardiovascular mortality risk in postmenopausal women. Am J Epidemiol 2002, 155(4):339-345.

36. Rosner W, Vesper H: Toward excellence in testosterone testing: a consensus statement. J Clin Endocrinol Metab 2010, 95(10):4542-4548.

37. Plymate SR, Tenover JS, Bremner WJ: Circadian variation in testosterone, sex hormone-binding globulin, and calculated non-sex hormone-binding globulin bound testosterone in healthy young and elderly men. $J$ Androl 1989, 10(5):366-371.

doi:10.1186/1471-2261-13-30

Cite this article as: Daka et al: Low sex hormone-binding globulin is associated with hypertension: a cross-sectional study in a Swedish population. BMC Cardiovascular Disorders 2013 13:30. 